\title{
Model-Based Library for Critical Dimension Metrology by CD-SEM
}

\author{
Y.B. Zou ${ }^{1}$, P. Zhang ${ }^{1}$, S.F. Mao ${ }^{2}$ and Z.J. Ding ${ }^{1}$ \\ 1. Hefei National Laboratory for Physical Sciences at Microscale and Department of Physics, University \\ of Science and Technology of China, 96 Jinzhai Road, Hefei, Anhui 230026, P.R. China \\ 2. School of Nuclear Science and Technology, University of Science and Technology of China, 96 \\ Jinzhai Road, Hefei, Anhui 230026, P.R. China
}

In integrated circuit industry, device metrology is crucial to the future development of semiconductor industry. Critical dimension scanning electron microscope (CD-SEM) is used as a tool for the linewidth measurement and critical dimension (CD) metrology. However, the signal intensity in a secondary electron image obtained by CD-SEM is influenced not only by geometry character of specimen but also by many experimental factors, leading to difficulty for linewidth measurement directly from the image contrast. A reasonable algorithm is thus necessary for the strict linewidth metrology and dimension control by CD-SEM.

Some works have been done on CD metrology and different algorithms for CD determination have been concerned [1,2]. Among them the model-based library (MBL) scheme was suggested to determine geometric parameters of a line [2]; the method compares the measured line scan profile in an SEM image directly with the simulation so as to find the best matched model input data in calculation. Construction of MBL by using a Monte Carlo (MC) simulation is considered to be an excellent approach because MC simulation of beam-sample interaction can take account of every possible physical factor that may affect signal intensity into the calculation; such obtained MBL relates the measured signal profiles with both the sample parameters and beam parameters.

The accuracy of MBL depends mostly on the accurate theoretical modeling of electron-solid interaction process; practically it also relates with number of parameters employed to describe beam and the sample property (e.g. material, geometry, charging and electric field). In this work we have used a state of art MC modeling for SEM image simulation: 1. the full Penn dielectric function formalism for the treatment of electron inelastic scattering and associated cascade secondary electron signal production $[3,4] ; 2$. a constructive solid geometry (CSG) modeling for a 3D structure with smooth surface and a finite element triangle mesh modeling for a rough/smooth surface [5]; 3. a ray-tracing technique for inhomogeneous specimen in composition and with a complex geometric structure, and, the space subdivision method for accelerating the calculation. The MC simulation model has been verified in our previous works in many aspects by comparing the simulated secondary electron yield, the secondary electron energy spectra, and SEM line-scan profiles with experimental data. The results of comparison have indicated that the MC simulation taking account of experimental factors for electron beam and sample should be reasonable and effective for MBL construction.

We are constructing MBL for resist line structure on Si wafer. Each sample is described with a set of geometric feature parameters for the structure, e.g. top CD (TCD), height (H), side-wall angle (SWA), root mean square (RMS) for Gaussian rough distribution. The beam parameters include primary energy, focusing condition (probe diameter, aperture angle and defocus). Sample charging condition will also be considered in future. While for rough surface sample, the line edge roughness (LER) and line width roughness (LWR) have become a critical issue when the linewidth for semiconductor devices shrink into 
a few tens of nanometers because it can degrade resolution and linewidth accuracy and cause fluctuation of transistor performance. Then the secondary electron line scan profiles have been simulated for the parameters within a range of practical use. Fig. 1(a) shows example line-scans by varying SWA for fixed height and top CD. Because the bottom CD is varied SWA then the line-scan shape and the measured line width changes with SWA. Fig. 1(b) shows the effect of surface roughness on line-scan profile and Fig. 1(c) illustrates the simulated SEM image of a line with surface roughness.

A software, CD-SEM Data Matching Tool, has been developed to search the best matched parameters in the library with line scan profiles taken from SEM image data. The cross section at one or several positions is firstly selected from an experimental SEM image to present line scan profiles, as shown in Fig. 2(a); then three best matched line-scans are obtained by searching MBL and by comparing the line shapes at edges, as shown in Fig. 2(b). The corresponding geometrical structure of the sample in the experiment is determined so long as the beam parameters are fully or partially known.

\section{References:}

[1] B. Bunday et al, Proc. SPIE 6152 (2006), p. 61520S-1.

[2] J.S. Villarrubia et al, Surf. Interface Anal. 37 (2005), p. 951.

[3] Z. J. Ding and R. Shimizu, Scanning 18 (1996), p. 92.

[4] S.F. Mao et al, J. Appl. Phys. 104 (2008), p. 114907-1.

[5] Y.G. Li et al, Scanning 35 (2013), p. 127.

[6] The authors acknowledge funding from the National Natural Science Foundation of China (Nos. 11274288 and 11204289), the National Basic Research Program of China (Nos. 2011CB932801 and 2012CB933702), Ministry of Education of China (No. 20123402110034) and "111" project (No. B07033), and Chinese Academy of Sciences (No. XXH12503-02-02-07).

(a)

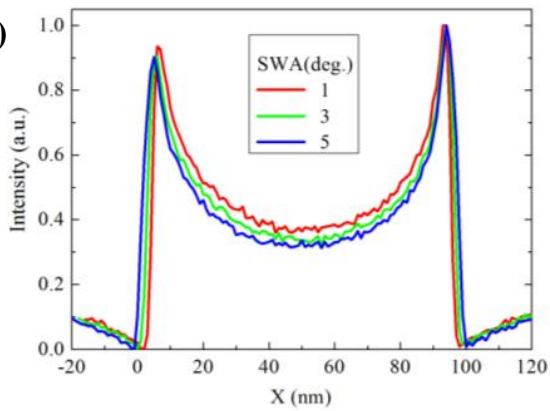

(b)

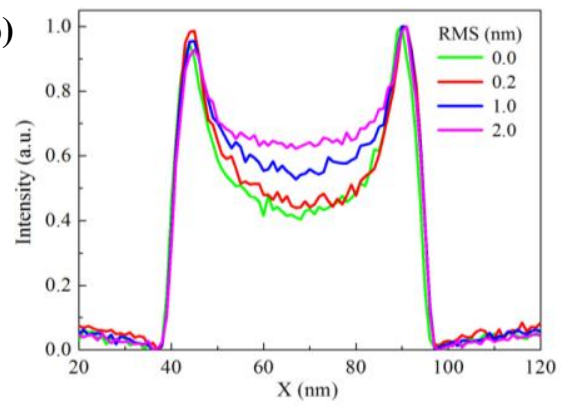

(c)

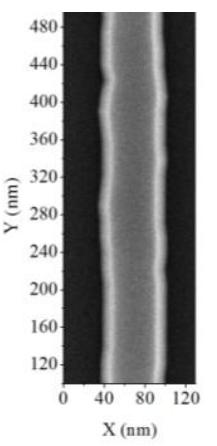

Figure 1. (a) Simulated secondary electron line scan profiles of $\mathrm{Au}$ line on $\mathrm{Si}$ substrate at the incident energy of $3 \mathrm{keV}$ for different SWA values and for $\mathrm{H}=50 \mathrm{~nm}$, TCD=90 nm. (b) Simulated line scan profiles of $\mathrm{Au}$ line with different RMS values for surface roughness. (c) Simulated SEM image of Au line with surface roughness on Si substrate.
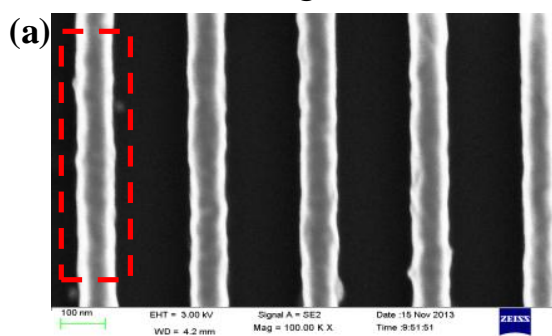

(b)

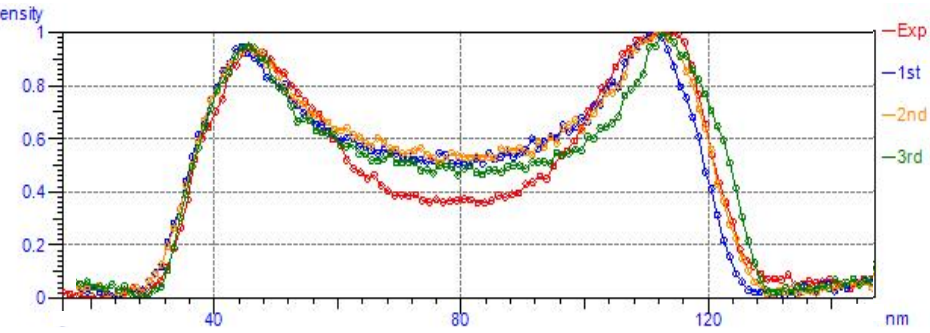

Figure 2. (a) Experimental SEM image where several selected cross sections in the red area gives line scan in (b): where the red line is experimental data, and others are best matched results from the library. 